\title{
Small bowel stricture following abdominal trauma
}

\author{
G.H. Welch and J.R. Anderson \\ Department of Surgery, Glasgow Royal Infirmary, Glasgow G31 2ER, UK.
}

\begin{abstract}
Summary: A case of small bowel stricture developing 2 months after blunt abdominal trauma from a lap seat belt is presented. The cause of the stricture was probably a small perforation sustained at the time of the injury but overlooked clinically. At operation an abscess around the terminal ileum was found. The patient made an uneventful recovery following resection of the strictured small bowel.
\end{abstract}

\section{Introduction}

The management of blunt abdominal trauma is noted for its difficulty in diagnosis. This can be due to masking of the abdominal insult by associated head, chest, and pelvic injuries. The small intestine, the most common hollow viscus involved has a $5-10 \%$ incidence of rupture (McBoyle et al., 1984). When perforation following trauma occurs the presentation is acute (Shuck \& Lowe, 1978), in contrast to the situation where the patient presents late with stricture formation (Marks et al., 1979).

We report a case of a small bowel stricture secondary to perforation which developed 2 months following blunt abdominal trauma. The abdominal injury was not detected at the initial presentation.

\section{Case report}

A 27 year old male was admitted in March 1984 to another hospital as an emergency following a road traffic accident in which the car he was driving struck a lamp post. He was wearing a seat belt. Examination on admission revealed a diffuse head injury with depressed conscious level, a displaced malar fracture, severe facial lacerations and enucleation of the left eye. There was no evidence of any other significant injury at that time. His lacerations were sutured and the fracture reduced and externally fixed. His conscious level gradually improved. He made an excellent recovery from his head and facial injuries but was readmitted on three further occasions with colicky abdominal pain. On each occasion no abnormality was detected clinically and his symptoms settled spontaneously.

Eight weeks after the accident he was admitted

Correspondence: G.H. Welch, F.R.C.S.

Accepted: 26 June 1985 under our care as an emergency with a further episode of severe abdominal pain. He had lost $15 \mathrm{~kg}$ in weight since his accident. Examination revealed evidence of intestinal obstruction and plain abdominal X-rays showed distension of both large and small bowel with multiple fluid levels suggesting obstruction in the descending colon. He was treated initially with intravenous fluid replacement and nasogastric suction. His symptoms and abdominal signs partially resolved and a barium enema was carried out. This was reported as normal. An antegrade small bowel enema was performed which showed marked dilatation of the distal small bowel, but the exact site of the obstruction was not demonstrated. In view of his continuing symptoms and radiological evidence of partial small bowel obstruction laparotomy was carried out.

At operation there was an inflammatory mass in the pelvis adherent to the anterior abdominal wall and the apex of the sigmoid loop. Two loops of jejunum and a hypertrophied segment of ileum entered the mass. The distal ileum was collapsed. The inflammatory mass was dissected from the sigmoid colon and jejunum and an abscess cavity which communicated with ileal lumen was found. The ileum at this point was strictured for a distance of $1 \mathrm{~cm}$. A short segment of distal ileum was resected with restoration of intestinal continuity by primary anastomosis. Histological examination of the resected specimen showed an acute inflammatory process with full thickness perforation of the bowel wall. He made an uneventful recovery following this procedure and was discharged from hospital 8 days later. At follow up 6 months later he was symptom free and had regained weight.

Review of the barium enema examination shows a stricture within the terminal ileum and an associated extraluminal gas filled structure corresponding to the abscess found at operation (Figure 1).

(C) The Fellowship of Postgraduate Medicine, 1985 


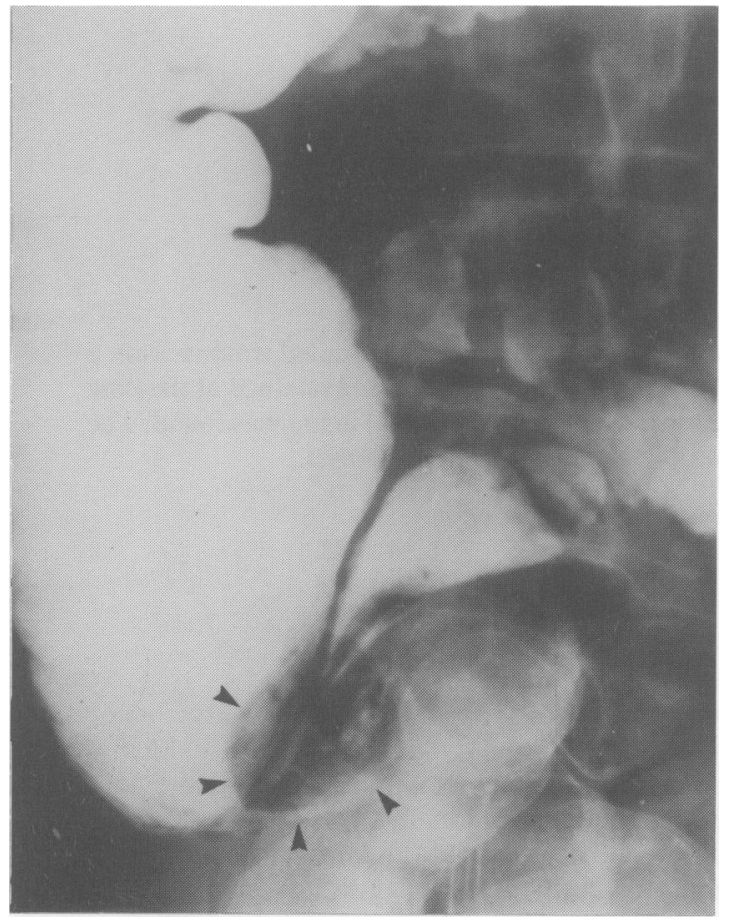

Figure 1 Barium enema examination showing tapered stricture in terminal ileum and gas filled abscess cavity (arrowed).

\section{References}

BRYNER, U.M., LONGERBEAM, J.K. \& REEVES, C.D. (1980). Posttraumatic ischaemic stenosis of the small bowel. Archives of Surgery, 115, 1039.

MCBOYLE, M.F., SCHILLER, W.R. \& HURT, A.V. (1984). Massive gastrointestinal bleeding following blunt abdominal trauma. Journal of Trauma, 24, 1057.

MARKS, C.G., NOLAN, D.J., PIRIS, J. \& WEBSTER, C.U. (1979). Small bowel strictures after blunt abdominal trauma. British Journal of Surgery, 66, 663.

\section{Discussion}

The late presentation of small bowel strictures following blunt abdominal trauma is uncommon. The aetiology is usually ischaemia secondary to mesenteric tears parallel and close to the involved segment, but occasionally direct injury to the bowel wall with subserosal haematoma formation may result in subsequent stenosis (Bryner et al., 1980). The proximal jejunum and terminal ileum are normally involved sites where the small bowel is relatively fixed (Marks $e t$ al., 1979).

The small bowel performation in the case presented was almost certainly sustained at the time of the initial injury, and was caused by the seat belt. When the perforation is limited it may become sealed by mucosa and omentum. Despite continuing symptoms, it was not until the patient presented with intestinal obstruction that the diagnosis was suspected. A high index of clinical suspicion is paramount when dealing with the victims of blunt abdominal trauma. When abdominal symptoms continue stricture formation should be considered and confirmed or excluded by a small bowel enema (Nolan \& Pantelidis, 1978). When the lesion is in the terminal ileum, barium enema examination may be of value in establishing the exact site of the lesion as was apparent in retrospect in the present case.

NOLAN, D.J. \& PANTELIDIS, N. (1978). Antegrade intubation barium examination of the small intestine. British Journal of Radiology, 51, 154.

SHUCK, J.M. \& LOWE, R.J. (1978). Intestinal disruption due to blunt abdominal trauma. American Journal of Surgery, 136, 668. 